\title{
Rezension „Die digitale Evolution moderner Großstädte“
}

\author{
Andreas Meier
}

Eingegangen: 12. Mai 2015 / Angenommen: 20. Mai 2015 / Online publiziert: 17. Juni 2015

(C) Springer Fachmedien Wiesbaden 2015

\section{Michael Jaekel und Karsten Bronnert}

Die digitale Evolution moderner Großstädte - Apps-basierte innovative Geschäftsmodelle für neue Urbanität

ISBN 978-3-658-00170-4, Springer Vieweg, Wiesbaden 2013, 190 S., 51,50€

Die beiden Autoren Michael Jaekel und Karsten Bronnert verfügen über mehrjährige Praxiserfahrung auf den Gebieten Strategieentwicklung, Geschäftsmodelle, ICT und Projektmanagement. Sie legen ein überschaubares Werk mit acht Kapiteln zur Evolution Smarter Cities vor.

Im Einführungskapitel wird aufgezeigt, welches Potenzial die Vernetzung aller Akteure in einer Smart City aufweist. Zudem wird plädiert, dass die urbanen Räumen vermehrt von der Kombination harter und weicher Standortfaktoren sowie der Nutzung von Wissenstechnologien abhängen. Als Treiber zur Modernisierung kann das Share-Modell dienen, bei dem soziale Nachbarschaften genutzt werden, um physische Ressourcen zu teilen.

Kapitel 2 ist betitelt mit: Moderne Städte sind smart. Nach der Erläuterung von Smartness wird die Smart City definiert. Als Charaktermerkmale gelten: Verwendung von Netzwerk-Strukturen, um die ökonomische und kulturelle Effizienz zu erhöhen und die soziale und urbane Entwicklung zu fördern. Besonderes Augenmerk kommt der sozialen Inklusion zu, da die Bewohnerinnen und Bewohner urbaner Räume von der digitalen Entwicklung nicht ausgeschlossen werden sollten. Eckpunkte der Handlungsfelder sind: Smart Economy, Smart People, Smart Governance, Smart Mobility, Smart Environment und Smart Living.

\footnotetext{
A. Meier $(\square)$

Universität Fribourg,

Fribourg, Schweiz

E-Mail: andreas.meier@unifr.ch 
Eine Vision moderner Großstädte wird in Kap. 3 anhand eines Apps-Oekosystems gegeben. Im Zentrum stehen digitale Dienstleistungen, die die städtische Ver- und Entsorgung unterstützen, so z. B. Verkehrssysteme auf Mietbasis oder Angebote von eHealth-Dienstleistungen oder Unterstützung im Alter etc.

Kapitel 4 widmet sich der Entwicklung der digitalen Technologien und erläutert das Potenzial mobiler Netzwerktechnologien sowie das Internet der Dinge. Im Vordergrund steht die Mensch-Maschine-Kommunikation sowie sogenannte Cyper Physical Systems. Darunter wird eine evolutionäre Weiterentwicklung der Kommunikation verstanden, um Verbundsysteme über Anwendungs- und Branchengrenzen hinweg zu ermöglichen. Als Schlüsseltechnologien werden InMemory-Techniken, Map/Reduce, Cloud Computing, MashUps, Open Data, Augmented Reality, Location-Based Services sowie Architekturen für Datenschutz und Datensicherheit angesehen.

Kapitel 5 erläutert das Geschäftsmodell smarter Großstädte. Hier wird ein SmartCity-Oekosystems beschrieben und es werden erste lauffähige Plattformen konkretisiert. Von Apps über Anwendungsfälle werden komplexe Anwendungswelten entwickelt. Zudem wird die Wertschöpfung aller Partner eines solchen Geschäftsmodells thematisiert. Konkrete Beispiele zu Apps für Smart Cities runden das Kapitel ab.

Das Kap. 6 beschreibt Umsetzungsszenarien für eine vernetzte urbane Mobilität. Die Politik wird als Motor und Treiber für künftige Mobilitätslösungen angesehen. Hier sind unterschiedliche Entwicklungsstufen zu unterscheiden: isolierte Apps als Einstieg in die Intermodalität, gebündelte Apps bis hin zu intermodalen Anwendungsszenarien.

Das letzte Kap. 7 beschreibt eine Vision vernetzter Gesundheitssysteme und schlägt eine entsprechende Webplattform vor, auf der alle Anspruchsgruppen und Gesundheitsleistungsanbieter interagieren. Im Zentrum soll eine bürgergeführte digitale Patientenakte stehen, die die Sicherung der Privatsphäre garantiert.

Die Autoren beschreiben ihre Vision einer Smart City und zeigen da und dort konkrete Beispiele auf. Zudem geben sie Handlungsempfehlungen für die Entscheidungsverantwortlichen solcher Initiativen. Auch wenn die vorgeschlagenen Geschäftsmodelle und die entsprechenden Apps-Architekturen noch nicht im Detail erprobt und umgesetzt wurden, gibt das Werk eine Anregung, wohin die urbane Mobilität hinführen könnte. Entsprechend richtet sich das Werk weniger an Informatiker oder Wirtschaftsinformatiker, sondern eher an Interessierte betreffend der Entwicklung urbaner Gebiete im digitalen Zeitalter. 